\title{
Low temperature properties of one-dimensional SU(4) Hubbard-like model at low concentration
}

\author{
Shi-Jian Gu \\ Zhejiang Institute of Modern Physics, Zhejiang University, Hangzhou,310027, China \\ You-Quan Li \\ Zhejiang Institute of Modern Physics, Zhejiang University, Hangzhou,310027, China \\ Institute for Physics, Augsburg University, D-86135 Augsburg, Germany \\ Zu-Jian Ying \\ Zhejiang Institute of Modern Physics, Zhejiang University, Hangzhou,310027, China
}

(Received:)

\begin{abstract}
On the basis of Bethe ansatz solution of one dimensional SU(4) Hubbardlike model, we study its thermodynamics properties by means of Yang-Yang thermodynamics Bethe ansatz. The Landé $g$ factor is taken into account so as to describe electrons with orbital degeneracy. The free energy at low temperature is given and the specific heat both in strong coupling and weak coupling limits are obtained.
\end{abstract}

\section{INTRODUCTION}

The study of integrable model has a long and rich history in condensed matter physics beginning with Bethe's solution of the one-dimensional Heisenberg Model and extending to nowadays when a variety of soluble models provide the paradigms that enrich much of our physical intuition. Recently there has been much interests in the studies on the $3 d$ electrons 
in transitional metal oxide日 because of the existence of orbital degree of freedom 2 目 in addition to the spin one. Since the SU(4) symmetry was pointed out 4 , there has been various studies, such as critical properties in photoemission spectra for the one-dimensional Mott insulator

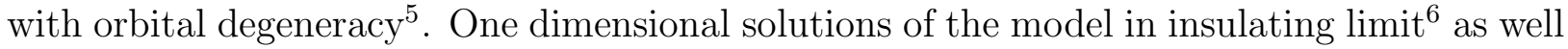
as the Hubbard-like model based on the SU(N) generalized Bethe ansatz equation 11 , but the physical meaning of the external field is ambiguous there because it is not associated with spin and orbital directly.

In present paper, we study the low-temperature thermodynamics of SU(4) Hubbardlike model for electrons with 2-fold orbital degeneracy at low concentration. The thermal equilibrium is discussed exactly on the basis of the known Bethe-ansatz equations by taking account of the Landé $g$ factor. Next section we first briefly exhibit the Bethe-ansatz equation under consideration, then discuss the thermodynamics of the model by considering string hypothesis. In Sec. III, we calculate the formal expressions of the thermodynamics quantities, such as free energy etc.. In Sec. IV, the case of low temperature is discussed extensively.

\section{THE MODEL AND ITS SPECTRUM IN THERMODYNAMICS LIMIT}

The model Hamiltonian reads

$$
\mathcal{H}=-t \sum_{i, a} \mathcal{P}\left(C_{i, a}^{+} C_{i+1, a}+C_{i+1, a}^{+} C_{i, a}\right) \mathcal{P}+U \sum_{i, a<a^{\prime}} n_{i, a} n_{i, a^{\prime}}
$$

where $i=1,2, \ldots, L$ identify the lattice site, and $a=1,2, \ldots, 4$ labels the four states of spin and orbitall . The $\mathcal{P}$ projects the Hilbert space onto the sector that the sites are only occupied by at most two electrons. The internal degree of freedom (11) is specified to spin

and orbital in present model. The Bethe-ansatz equation for the spectrum was suggested目 as

$$
e^{i k_{j} L}=\prod_{b=1}^{M} \frac{\sin k_{j}-\lambda_{b}+i \eta}{\sin k_{j}-\lambda_{b}-i \eta}
$$




$$
\begin{aligned}
\prod_{l=1}^{N} \frac{\lambda_{a}-\sin k_{l}+i \eta}{\lambda_{a}-\sin k_{l}-i \eta} & =-\prod_{b=1}^{M} \frac{\lambda_{a}-\lambda_{b}+i 2 \eta}{\lambda_{a}-\lambda_{b}-i 2 \eta} \prod_{c=1}^{M^{\prime}} \frac{\mu_{c}-\lambda_{a}+i \eta}{\mu_{c}-\lambda_{a}-i \eta} \\
& \prod_{b=1}^{M} \frac{\mu_{a}-\lambda_{b}+i \eta}{\mu_{a}-\lambda_{b}-i \eta}=-\prod_{c=1}^{M^{\prime}} \frac{\mu_{a}-\mu_{c}+i 2 \eta}{\mu_{a}-\mu_{c}-i 2 \eta} \prod_{d=1}^{M^{\prime \prime}} \frac{\nu_{d}-\mu_{a}+i \eta}{\nu_{d}-\mu_{a}-i \eta} \\
& \prod_{b=1}^{M^{\prime}} \frac{\nu_{a}-\mu_{b}+i \eta}{\nu_{a}-\mu_{b}-i \eta}=-\prod_{c=1}^{M^{\prime \prime}} \frac{\nu_{a}-\mu_{c}+i 2 \eta}{\nu_{a}-\mu_{c}-i 2 \eta}
\end{aligned}
$$

where $\eta=U / 4 t$. Unlike the conventional $\mathrm{SU}(2)$ Hubbard model the $\mathrm{SU}(4)$ generalization (2) of Lieb-Wu solution $\mathrm{g}$ is valid at low temperature and low concentration 19,11 .

For ground state (i.e., at zero temperature), the $k, \lambda, \mu, \nu$ are real roots of the Bethe ansatz equation (2). However, for the excited state, they can be complex roots. We will not take account of the complex roots in the charge space $k$ for repulsive interaction 12 . The complex root of $\lambda, \mu, \nu$ are always form a "bound state" with several same kind of rapidity which arise from the consistency of both hand side of the Bethe ansatz equations. The complex roots are defined as

$$
\begin{array}{cl}
\Lambda_{a}^{n j}=\lambda_{a}^{n}+(n+1-2 j) i \eta, & j=1,2 \ldots, n, \\
U_{b}^{m j}=\mu_{b}^{m}+(m+1-2 j) i \eta, & j=1,2 \ldots, m, \\
V_{c}^{l j}=\nu_{c}^{l}+(l+1-2 j) i \eta, & j=1,2 \ldots, l,
\end{array}
$$

up to the order $O\left(e^{-L}\right)$ which vanishes in the thermodynamic limit. Substituting those strings into Eq. (2) and taking the logarithm of it, we get

$$
\begin{aligned}
2 \pi I_{j} & =k_{j} L+2 \sum_{a n} \tan ^{-1}\left(\frac{\sin k_{j}-\lambda_{a}^{n}}{n \eta}\right), \\
2 \pi J_{a}^{n} & =2 \sum_{l} \tan ^{-1}\left(\frac{\lambda_{a}^{n}-\sin k_{l}}{n \eta}\right)-2 \sum_{b m l} A_{n m l} \tan ^{-1}\left(\frac{\lambda_{a}^{n}-\lambda_{b}^{m}}{l \eta}\right)-2 \sum_{c l t} B_{n l t} \tan ^{-1}\left(\frac{\mu_{c}^{l}-\lambda_{a}^{n}}{t \eta}\right), \\
2 \pi K_{a}^{n} & =2 \sum_{b l t} B_{n l t} \tan ^{-1}\left(\frac{\mu_{a}^{n}-\lambda_{b}^{l}}{t \eta}\right)-2 \sum_{c l t} A_{n l t} \tan ^{-1}\left(\frac{\mu_{a}^{n}-\mu_{c}^{l}}{t \eta}\right)-2 \sum_{d l t} B_{n l t} \tan ^{-1}\left(\frac{\nu_{d}^{l}-\mu_{a}^{n}}{t \eta}\right), \\
2 \pi Q_{a}^{n} & =2 \sum_{b l t} B_{n l t} \tan ^{-1}\left(\frac{\nu_{a}^{n}-\mu_{b}^{l}}{t \eta}\right)-2 \sum_{c l t} A_{n l t} \tan ^{-1}\left(\frac{\nu_{a}^{n}-\nu_{c}^{l}}{t \eta}\right) .
\end{aligned}
$$

where 


$$
\begin{aligned}
& A_{n m p}=\left\{\begin{array}{l}
1, \text { for } p=m+n,|m-n|(\neq 0), \\
2, \text { for } p=n+m-2, n+m-4, \cdots,|n-m|+2, \\
0, \text { otherwise }
\end{array}\right. \\
& B_{n l t}= \begin{cases}1, & \text { for } t=n+l-1, n+l-3, \ldots,|n-l|+1, \\
0, & \text { otherwise. }\end{cases}
\end{aligned}
$$

Noe we consider the question in the thermodynamic limit $N, L, M, M^{\prime}, M^{\prime \prime} \rightarrow \infty$ with a fixed concentration $D=N / L$ by introducing the distribution of roots and holes for $k, \lambda, \mu, \nu$ respectively:

$$
\begin{aligned}
\frac{1}{L} \frac{d I(k)}{d k} & =\rho(k)+\rho^{h}(k), \\
\frac{1}{L} \frac{d J_{n}(\lambda)}{d \lambda} & =\sigma_{n}(\lambda)+\sigma_{n}^{h}(\lambda), \\
\frac{1}{L} \frac{d K_{n}(\mu)}{d \mu} & =\omega_{n}(\mu)+\omega_{n}^{h}(\mu), \\
\frac{1}{L} \frac{d Q_{n}(\nu)}{d \nu} & =\tau_{n}(\nu)+\tau_{n}^{h}(\nu) .
\end{aligned}
$$

We obtain the following coupled integral equations.

$$
\begin{aligned}
\rho+\rho^{h} & =\frac{1}{2 \pi}+\sum_{n} \cos k \int K_{n}(\sin k-\lambda) \sigma_{n}(\lambda) d \lambda \\
\sigma_{n}+\sigma_{n}^{h} & =\int K_{n}(\lambda-\sin k) \rho(k) d k-\sum_{m l} A_{n m l} \int K_{l}\left(\lambda-\lambda^{\prime}\right) \sigma_{m}\left(\lambda^{\prime}\right) d \lambda^{\prime}+\sum_{l t} B_{n l t} \int K_{t}(\lambda-\mu) \omega_{l}(\mu) d \mu \\
\omega_{n}+\omega_{n}^{h} & =\sum_{l t} B_{n l t} \int K_{t}(\mu-\lambda) \sigma_{l}(\lambda) d \lambda-\sum_{l t} A_{n l t} \int K_{t}\left(\mu-\mu^{\prime}\right) \omega_{l}\left(\mu^{\prime}\right) d \mu^{\prime}+\sum_{l t} B_{n l t} \int K_{t}(\mu-\nu) \tau_{l}(\nu) d \nu \\
\tau_{n}+\tau_{n}^{h} & =\sum_{l t} B_{n l t} \int K_{t}(\nu-\mu) \omega_{l}(\mu) d \mu-\sum_{l t} A_{n l t} \int K_{t}\left(\nu-\nu^{\prime}\right) \tau_{l}\left(\nu^{\prime}\right) d \nu^{\prime}
\end{aligned}
$$

where $K_{n}(x)=\pi^{-1} n \eta /\left(n^{2} \eta^{2}+x^{2}\right)$, and the integration limits are defined by

$$
\begin{aligned}
\frac{N}{L} & =\int_{-Q_{0}}^{Q_{0}} \rho(k) d k, \\
\frac{M}{L} & =\sum_{n} n \int_{-B_{1}}^{B_{1}} \sigma_{n}(\lambda) d \lambda, \\
\frac{M^{\prime}}{L} & =\sum_{n} n \int_{-B_{2}}^{B_{2}} \omega_{n}(\mu) d \mu, \\
\frac{M^{\prime \prime}}{L} & =\sum_{n} n \int_{-B_{3}}^{B_{3}} \tau_{n}(\nu) d \nu .
\end{aligned}
$$




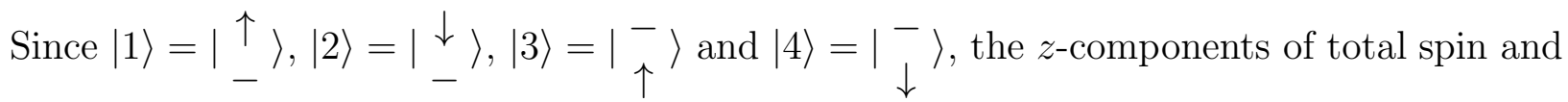
orbital are given by,

$$
\begin{aligned}
\frac{S_{z}}{L} & =\frac{1}{2} \int \rho d k+\sum_{n} n \int \omega_{n} d \mu-\sum_{n} n \int \sigma_{n} d \lambda-\sum_{n} n \int \tau_{n} d \nu \\
\frac{T_{z}}{L} & =\frac{1}{2} \int \rho d k-\sum_{n} n \int \omega_{n} d \mu .
\end{aligned}
$$

The energy is given by

$$
\frac{E}{L}=-2 t \int \cos k \rho(k) d k
$$

and the magnetization by

$$
M_{z}=g_{s} S_{z}+g_{t} T_{z}
$$

where $g_{s}, g_{t}$ are Landé $g$ factors that we know $g_{s}=2, g_{t}=1$, which was ignored in previous literature because it was not able to be related to spin-orbital model.

\section{THERMAL EQUILIBRIUM}

Yang and Yang 13 first introduced a definition of entropy in terms of distribution of roots and holes for Bethe-ansatz solvable systems. This method was recently carefully compared with the transfer matrix approach, the results of both approach are shown in agreement with each other 4 . In order to obtain thermal equilibrium at finite temperature, we should maximize the free energy $\Omega=E+E_{J}-T \mathcal{S}-\mathcal{A} N$ where $E_{J}=-\mu_{0} H M_{z}$ is the Zeemann energy due to the external magnetic field and $\mathcal{A}$ is the chemical potential. The entropy is given by

$$
\begin{aligned}
\frac{\mathcal{S}}{L}= & \int\left[\left(\rho+\rho^{h}\right) \ln \left(\rho+\rho^{h}\right)-\rho \ln \rho-\rho^{h} \ln \rho^{h}\right] d k \\
& +\sum_{n} \int\left[\left(\sigma_{n}+\sigma_{n}^{h}\right) \ln \left(\sigma_{n}+\sigma_{n}^{h}\right)-\sigma_{n} \ln \sigma_{n}-\sigma_{n}^{h} \ln \sigma_{n}^{h}\right] d \lambda \\
& +\sum_{n} \int\left[\left(\omega_{n}+\omega_{n}^{h}\right) \ln \left(\omega_{n}+\omega_{n}^{h}\right)-\omega_{n} \ln \omega_{n}-\omega_{n}^{h} \ln \omega_{n}^{h}\right] d \mu \\
& +\sum_{n} \int\left[\left(\tau_{n}+\tau_{n}^{h}\right) \ln \left(\tau_{n}+\tau_{n}^{h}\right)-\tau_{n} \ln \tau_{n}-\tau_{m}^{h} \ln \tau_{n}^{h}\right] d \nu .
\end{aligned}
$$


We obtain the Helmholtz free energy from the condition $\delta \Omega=0$ that

$$
F=-\frac{T L}{2 \pi} \int \ln \left[1+e^{-\epsilon}\right] d k
$$

where $\epsilon$ should be solved from the following equations,

$$
\begin{aligned}
\epsilon= & -\frac{1}{T}\left[2 t \cos k+\mathcal{A}+\frac{3}{2} \mu_{0} H\right]-\sum_{n} \int K_{n}(\lambda-\sin k) \ln \left[1+e^{-\theta_{n}(\lambda)}\right] d \lambda, \\
\theta_{n}= & 2 \mu_{0} n H / T-\int \cos k K_{n}(\sin k-\lambda) \ln \left[1+e^{-\epsilon(k)}\right] d k \\
& +\sum_{l t} A_{n l t} \int K_{t}\left(\lambda-\lambda^{\prime}\right) \ln \left[1+e^{-\theta_{l}\left(\lambda^{\prime}\right)}\right] d \lambda^{\prime}-\sum_{l t} B_{n l t} \int K_{t}(\mu-\lambda) \ln \left[1+e^{-\zeta_{l}(\mu)}\right] d \mu, \\
\zeta_{n}= & -\mu_{0} n H / T-\sum_{l t} B_{n l t} \int K_{t}(\lambda-\mu) \ln \left[1+e^{-\theta_{l}(\lambda)}\right] d \lambda \\
& +\sum_{l t} A_{n l t} \int K_{t}\left(\mu-\mu^{\prime}\right) \ln \left[1+e^{-\zeta_{l}\left(\mu^{\prime}\right)}\right] d \nu^{\prime}-\sum_{l t} B_{n l t} \int K_{t}(\nu-\mu) \ln \left[1+e^{-\xi_{l}(\nu)}\right] d \nu, \\
\xi_{n}= & 2 \mu_{0} n H / T-\sum_{l t} B_{n l t} \int K_{t}(\mu-\nu) \ln \left[1+e^{-\zeta_{l}(\mu)}\right] d \mu \\
& +\sum_{l t} A_{n l t} \int K_{t}\left(\nu-\nu^{\prime}\right) \ln \left[1+e^{-\xi_{l}\left(\nu^{\prime}\right)}\right] d \nu^{\prime} .
\end{aligned}
$$

In getting the above expressions, we have introduced notations:

$$
e^{\epsilon}=\frac{\rho^{h}}{\rho}, \quad e^{\theta_{n}}=\frac{\sigma_{n}^{h}}{\sigma_{n}}, \quad e^{\zeta_{n}}=\frac{\omega_{n}^{h}}{\omega_{n}}, e^{\xi_{n}}=\frac{\tau_{n}^{h}}{\tau_{n}} .
$$

\section{THE CASE AT LOW TEMPERATURE}

At very low temperature, the density of roots undergo a slight modification from that of the ground state, and the right hand side of Eq. (14) are approximately zero below Fermi surface. Then we have

$$
\begin{aligned}
& \epsilon=-\frac{1}{T}\left[2 t \cos k+\mathcal{A}+\frac{3}{2} \mu_{0} H\right]+\int_{-B_{1}}^{B_{1}} K_{1}(\lambda-\sin k) \theta(\lambda) d \lambda \\
& \theta=2 \mu_{0} H / T+\int_{-Q_{0}}^{Q_{0}} \cos k K_{1}(\sin k-\lambda) \epsilon(k) d k-\int_{-B_{1}}^{B_{1}} K_{2}\left(\lambda-\lambda^{\prime}\right) \theta\left(\lambda^{\prime}\right) d \lambda^{\prime}+\int_{-B_{2}}^{B_{2}} K_{1}(\mu-\lambda) \zeta(\mu) d \mu \\
& \zeta=-\mu_{0} H / T+\int_{-B_{1}}^{B_{1}} K_{1}(\lambda-\mu) \theta(\lambda) d \lambda-\int_{-B_{2}}^{B_{2}} K_{2}\left(\mu-\mu^{\prime}\right) \zeta\left(\mu^{\prime}\right) d \mu^{\prime}+\int_{-B_{3}}^{B_{3}} K_{1}(\nu-\mu) \xi(\nu) d \nu \\
& \xi=2 \mu_{0} H / T+\int_{B_{2}}^{B_{2}} K_{1}(\mu-\nu) \zeta(\mu) d \mu-\int_{-B_{3}}^{B_{3}} K_{2}\left(\nu-\nu^{\prime}\right) \xi\left(\nu^{\prime}\right) d \nu^{\prime} .
\end{aligned}
$$


It is plausible to take $B_{1}, B_{2}, B_{3}=\infty$ in our present case. By making a Fourier transform of the second through fourth equation of Eq. (15), we have

$$
\begin{aligned}
& \tilde{\theta}=2 \tilde{h}+\frac{1}{2 \pi} \int_{-Q_{0}}^{Q_{0}} \cos k e^{-\eta|w|-i w \sin k} \epsilon(k) d k-e^{-2 \eta|w|} \tilde{\theta}+e^{-\eta|w|} \tilde{\zeta} \\
& \tilde{\zeta}=-\tilde{h}+e^{-\eta|w|} \tilde{\theta}-e^{-2 \eta|w|} \tilde{\zeta}+e^{-\eta|w|} \tilde{\xi} \\
& \tilde{\xi}=2 \tilde{h}+e^{-\eta|w|} \tilde{\zeta}-e^{-2 \eta|w|} \tilde{\xi}
\end{aligned}
$$

where $\tilde{h}=\mu_{0} H \delta(w) / T$. It is not difficult to derive an integral equation for $\epsilon$ :

$$
\epsilon=-[2 t \cos k+\mathcal{A}] / T+\frac{1}{\eta} \int_{-k_{F}}^{k_{F}} \cos k^{\prime} \epsilon\left(k^{\prime}\right) R_{3}\left(\frac{\sin k-\sin k^{\prime}}{\eta}\right) d k
$$

where $k_{F}$ is the Fermi momentum and

$$
R_{n}(x)=\frac{1}{2 \pi} \int_{-\infty}^{\infty} \frac{\sinh (n w)}{\sinh (4 w)} e^{i w x-|w|} d w
$$

In the absence of external field $(H=0)$, Eq.(17) is rigorous. When the external field is weak, it is also plausible because the changes on the integral interval of spin and orbit rapidity is of order of $1 / L$ in the thermodynamic limit. Moreover, even if we consider the contribution of the densities outside the Fermi surface, it is of order $e^{-1 / T}$. Clearly the presence of external field has no explicit effect on the ratio of density of charge roots and holes at low temperature. We can also infer from Eq.(17) and Eq. (10) there will be two critical fields $H_{c}^{1}, H_{c}^{2}$ which can cause phase transition on the magnetization of the system.

Once $\epsilon$ is solved, the free energy at low temperature can be evaluated by Eq.(12). Though an explicit expression can not be obtained from Eq. (17) in general case, it is easier for numerical calculation. Define the density of roots at low temperature as $\rho=\rho_{0}+\rho_{1}$, where $\rho_{1}$ is a slight deviation from the density of the ground state $\rho_{0}$, we can obtain

$$
\rho=\rho_{0}\left(1-e^{\epsilon}\right)-\frac{1}{\pi} \int \frac{\cos k \eta \sigma_{0} e^{\theta} d \lambda}{\eta^{2}+(\sin k-\lambda)^{2}}
$$

Substituting $\epsilon(k)$ of Eq. (17) into Eq. (12), we obtain

$$
F=\frac{T L}{2 \pi} \int \epsilon d k-\frac{T L}{2 \pi} \int e^{\epsilon} d k .
$$


Clearly when $T \rightarrow 0$ the free energy is just the ground state energy except for the additional chemical potential term $-\mathcal{A} L$ and the $\epsilon(k)$ just play a role of so called dressed energy.

\section{Strong coupling limit}

In strong coupling limit $\eta \rightarrow \infty$, we have

$$
\epsilon=-[2 t \cos k+\mathcal{A}] / T
$$

Since we consider the problem at low concentration, the Fermi surface is much less than $\pi$. Then we can rewrite Eq. (21) as

$$
\epsilon=\left[t k^{2}-\kappa\right] / T
$$

where $\kappa=2 t+\mathcal{A}$. And the distribution of the roots becomes

$$
\rho=\frac{1}{2 \pi} \frac{1}{1+e^{\left[t k^{2}-\kappa\right] / T}}
$$

with condition $\int_{-k_{F}}^{k_{F}} \rho(k) d k=D$, and when $T=0$, it gives $k_{F}=\sqrt{\kappa_{0} / t}$. We obtain

$$
\kappa=\kappa_{0}\left[1-\frac{\pi^{2} T^{2}}{24 \kappa_{0}^{2}}\right]^{-2},
$$

where $\kappa_{0}=t \pi^{2} D^{2}$ is the value of $\kappa$ at zero temperature. Obviously the system's energy becomes

$$
\frac{E}{L} \doteq \frac{\kappa_{0}^{3 / 2}}{3 \pi \sqrt{t}}\left[1+\frac{\pi^{2} T^{2}}{4 \kappa_{0}^{2}}\right]
$$

The specific heat and the entropy of the system are calculated as

$$
C_{V}=\mathcal{S}=\frac{T}{6 t D}
$$

which is Fermi-liquid like.

\section{Weak coupling limit}

In weak coupling $\eta \rightarrow 0$, Eq.(17) can be solved exactly. In mathematics we have

$$
\lim _{\eta \rightarrow 0} \frac{\sinh (3 \eta)}{\sinh (4 \eta)} e^{-\eta}=\frac{3}{4},
$$


Then the Eq. (18) is proportional to $\delta$-function. The $\epsilon(k)$ has the form

$$
\epsilon=-[8 t \cos k+4 \mathcal{A}] / T
$$

So we can repeat the steps in the section of strong coupling limit. The specific heat and the entropy become

$$
C_{V}=\mathcal{S}=\frac{T}{96 t D}
$$

Clearly it is still Fermi-liquid like. We can infer at low temperature the specific heat of the system is Fermi-liquid like regardless the value of coupling constant. The difference is the coefficient.

\section{Magnetic properties}

In the presence of external field, the densities of spin and orbit rapidities will change. We rewrite them as

$$
\begin{gathered}
\sigma=\sigma_{0}\left(1-e^{\theta}\right), \\
\omega=\omega_{0}\left(1-e^{\zeta}\right), \\
\tau=\tau_{0}\left(1-e^{\xi}\right) .
\end{gathered}
$$

where $\theta, \zeta, \xi$ are all negative and proportional to $H / T$. Then the magnetization of the system has the form

$$
M_{z} / L=\frac{3}{2} \int \rho d k+\int \omega d \mu-2 \int \sigma d \lambda-2 \int \tau d \nu
$$

Concerning $M=3 N / 4, M^{\prime}=N / 2, M^{\prime \prime}=N / 4$ at the ground state, we obtain

$$
M_{z} / L=2 \int \sigma_{0} e^{\theta} d \lambda-\int \omega_{0} e^{\zeta} d \mu+2 \int \tau_{0} e^{\xi} d \nu
$$

and the susceptibility in the weak external field is

$$
\chi=\frac{3 \mu_{0}}{T} \int \sigma_{0} e^{\theta} d \lambda-\frac{\mu_{0}}{T} \int \omega_{0} e^{\zeta} d \mu+\frac{3 \mu_{0}}{T} \int \tau_{0} e^{\xi} d \nu
$$

However, if the external field is strong enough so that there are no spin and orbit flipped down. That is $M=M^{\prime}=M^{\prime \prime}=0$ and the densities of $\lambda, \mu, \nu$ are all zero. Then we have 


$$
\rho+\rho^{h}=\rho\left(1+e^{\epsilon}\right)=\frac{1}{2 \pi}
$$

where $\epsilon$ is given by

$$
\begin{aligned}
\epsilon & =-\frac{1}{T}\left[2 t \cos k+\mathcal{A}+\frac{3}{2} \mu_{0} H\right], \\
2 \mu_{0} H_{c} / T & =-\int \cos k K_{1}(\sin k-\lambda) \epsilon d k .
\end{aligned}
$$

The $H_{c}$ is the maximal value of $\left\{H_{c}^{1}, H_{c}^{2}\right\}$ mentioned above. At large coupling constant the value of $H_{c}$ scales like $1 / \eta$. Clearly, in the limit $\eta \rightarrow \infty$, a small value of $H$ can flip all spin and orbit to upward. The density of charge roots becomes

$$
\rho=\frac{1}{2 \pi} \frac{1}{1+e^{-[2 t \cos k+2 t] / T}}
$$

which takes the value of $1 / 2 \pi$ at the ground state. The density of charge roots in strong coupling limit takes the same form as that in sufficient strong external field.

\section{SUMMARY AND ACKNOWLEDGMENTS}

In the above, we discussed one-dimensional SU(4) Hubbard-like Model in the thermodynamic limit, which is reliable at low temperature and low concentration. We studied the property of the thermal equilibrium and obtained the equilibrium equations. In the low temperature limit, the T-dependent density of roots and free energy, specific heat both in strong and weak coupling limit, as well as magnetic properties of the system are calculated.

This work is supported by NSFC No.1-9975040, trans-century project and EYF of China Education Ministry. Y.Q.Li acknowledges the support of AvH-stiftung. S.J.Gu acknowledges interesting discussions with J.H.Dai and D.Yang. 


\section{REFERENCES}

${ }^{1}$ Y. Tokura and N. Nagaosa, Science 288, 462 (2000).

${ }^{2}$ D. B. McWhan, T. M. Rice, and J. P. Remeika, Phys. Rev. Lett 23, 1384 (1969); C. Castellani, C. R. Natoli, and J. Ranninger, Phys. Rev. B18 4945, 4967 and 5001 (1978); R. E. Word, S. A. Werner, W. B. Yelon, J. M. Honig, and S. Shivashankar, Phys. Rev. B23, 3533 (1981).

${ }^{3}$ H. F. Pen, J. Brink, D. I. Komomskii, and G. A. Sawatzky, Phys. Rev. Lett. 78, 1323 (1996); B. J. Sternlieb, J. P. Hill, and U. C. Wildgruber, Phys. Rev. Lett. 76, 2196 (1996); Y. Moritomo, A. Asamitsu, H. Kuwahara, and Y. Tokura, Nature 380, 141 (1996).

${ }^{4}$ Y. Q. Li, M. Ma, D. N. Shi, and F. C. Zhang, Phys. Rev. Lett. 81, 3527 (1998).

${ }^{5}$ T. Fuji, Y. Tsukamoto, and N. Kawawami, J. Phys. Soc. Jpn., 68, 151 (1999).

${ }^{6}$ Y. Q. Li, M. Ma, D. N. Shi, and F. C. Zhang, Phys. Rev. B60, 12781 (1999).

${ }^{7}$ Y. Q. Li, S. J. Gu, Z. J. Ying, and U. Eckern, Phys. Rev. B62, 4866 (2000).

${ }^{8}$ T.C. Choy, Phys. Lett. 80 A, 49 (1980).

${ }^{9}$ E. H. Lieb and F.Y. Wu, Phys. Rev. Lett. 25, 1445 (1968).

${ }^{10}$ T. C. Choy and F. D. M. Haldane, Phys. Lett. A 90, 83 (1982).

${ }^{11}$ H. Frahm and A. Schadschneider, The Hubbard model: Its Physics and Mathematical Physics Eds. D. Baeriswyl et al., (Plenum Press, New York 1995) pp. 21. P. Schlottmann, Int. J. Mod. Phys. B 11, 355 (1997).

${ }^{12}$ M. Takahashi, Prog. Thero. Phys. 52, 103 (1974).

${ }^{13}$ C. N. Yang, and C. P. Yang, J. Math. Phys. 10, 1115 (1969)

${ }^{14}$ T. Deguchi, F.H.L. Essler, F.Gohmann, A. Klumper, V.E. Korepin, K. Kusakabe, Phys. Rep. 331,197 (2000). 